\title{
Optical analysis of magnetic microstructures
}

\section{Ruben Piepgras, Sebastian Michlmayr, Bernhard Zagar}

Ruben Piepgras, Sebastian Michlmayr, Bernhard Zagar, "Optical analysis of magnetic microstructures," Proc. SPIE 11144, Photonics and Education in Measurement Science 2019, 111440X (17 September 2019); doi:

$10.1117 / 12.2530795$

SDIE Event: Joint TC1 - TC2 International Symposium on Photonics and Education in Measurement Science 2019, 2019, Jena, Germany 


\title{
Optical Analysis of Magnetic Microstructures
}

\author{
Ruben Piepgras ${ }^{\mathrm{a}}$, Sebastian Michlmayr ${ }^{\mathrm{a}}$, and Bernhard Zagar ${ }^{\mathrm{a}}$ \\ anstitute for Measurement Technology, Johannes Kepler University Linz, \\ Altenberger Straße 69, 4040 Linz, Austria
}

\begin{abstract}
Encoding information using magnetic microstructures is utilised e.g. in magnetic stripe cards or banknotes. In order to analyse these structures quickly and non-destructively a magneto-optical setup based on the Faraday effect can be used. The Faraday effect states that the plane of polarisation of polarised light is rotated when it passes a Faraday-active material and is subjected to an external magnetic field. Measuring the change of intensity of light passing a set of polarisers allows the calculation of the change of the polarisation angle, which in turn allows drawing conclusions on the magnitude of the external magnetic field.

A first setup yielded very good results in the high-speed analysis of patterns with a structure size of about $50 \mu \mathrm{m}$. However, the setup's low amplitude resolution only allowed qualitative measurements. In order to find the limitations of this measurement principle with respect to amplitude, temporal, and spatial resolution as well as their interdependence a new setup was purpose-built for characterisation. Its components were examined closely and various methods of signal enhancement were evaluated.

The measurements displayed long- and short-term temporal as well as spatial dependencies. The subsequent enhancement of the signal's amplitude resolution came with a loss in temporal or spatial resolution and viceversa. The interdependence of amplitude, spatial, and amplitude resolution was characterised further and from this a generalised description of their lower bound for a given set of parameters was derived. This should serve as an estimate of the feasibility as well as a build guideline of a similar setup.
\end{abstract}

Keywords: Non-Destructive Testing, Magnetic Microstructures, Faraday Rotation Magnetometry.

\section{INTRODUCTION}

Magnetic microstructures are a useful tool to encode information. They are used e.g. in banknotes as a means of anti-counterfeiting. Here, stripes measuring a couple of millimetres in width with specific magnetic patterns are woven in the banknotes' fibres. These stripes are produced in a roll-to-roll process where a polyethylene foil with a thickness of $50 \mu \mathrm{m}$ is moved at a speed of up to $3 \mathrm{~m} \mathrm{~s}^{-1}$. Upon this foil the magnetic patterns as well as additional layers are printed. The magnetic patterns have a structure size of approximately $60 \mu \mathrm{m}$ and a layer thickness of $500 \mathrm{~nm}$. The differentiating criterion within the pattern are the printing materials or rather their magnetic properties. As of now two materials are used with remanent magnetisations of $30 \mathrm{mT}$ and around $300 \mathrm{mT}$ respectively. The additional layers are non-magnetic and purely serve masking purposes against simple optical pattern analysis.

In order to assure the possibility of a timely intervention an in-line quality assessment is required. The method proposed in this contribution is a two-step process which consists of the magnetisation of the pattern and the subsequent measurement with a magneto-optical setup based on the Faraday Effect - a so-called Faraday Rotation Magnetometer (FRM). Due to the non-transparent nature of the stripes the FRM needs to be adapted for usage from one side.

$\operatorname{In}^{1}$ Egger et al. presented a setup that was built around a highly sensitive mirrored Faraday crystal. The light incidence upon this crystal was non-orthogonal so that the reflected light could directly be measured without further use of mirrors. The data acquisition was carried out by a high-speed line-scan camera and was followed

Further author information: (Send correspondence to R.P.)

R.P.: E-mail: ruben.piepgras@jku.at, Telephone: +43 (0)732 24685928

B.Z.: E-mail: bernhard.zagar@jku.at, Telephone: +43 (0)732 24685921

Photonics and Education in Measurement Science 2019, edited by Maik Rosenberger,

Paul-Gerald Dittrich, Bernhard Zagar, Proc. of SPIE Vol. 11144, 111440X

(C) 2019 SPIE · CCC code: 0277-786X/19/\$21 - doi: 10.1117/12.2530795 
by a data processing unit. The need for a resolution of around $60 \mu \mathrm{m}$ at the foils maximum speed as well as hour-long recording times entailed poor illumination at the camera and high sustained data-rates in the data processing unit amongst many others. This resulted in extreme demands on many parts of the setup. These difficulties notwithstanding, it was possible to record meaningful data at the required speed and for several hours of continuous measurement. The recorded images made it possible to distinguish the different elements of the magnetic pattern.

However, these results have to be considered qualitatively as it is not possible to differentiate between areas with materials with more similar coercitivities. This makes it an unsuitable device for any meaningful quantitative measurements. Nonetheless, it remains of interest if a differently built FRM might yet be suitable. To this end and building on the underlying theory, a close examination of a generalised form of the FRM and its components was carried out. Additionally, various possible steps of signal enhancement were analysed closely. This allowed to ascertain the limitations of the measurement principle regarding amplitude, spatial, and temporal resolution as well as their interdependence.

\section{THEORETICAL BACKGROUND}

The Faraday effect describes how a magnetic field affects linearly polarised light passing a Faraday-active material - a so-called Faraday crystal. When linearly polarised light passes a Faraday crystal, which is subjected to an external magnetic field in the direction of light propagation, the angle of the light's polarisation plane rotates. Due to the crystal's thickness of only a few $\mu \mathrm{m}$ the magnetic field is assumed to be constant along it. The Faraday effect then states ${ }^{2,3}$ that

$$
\beta=H \cdot d \cdot V,
$$

where $\beta$ is the rotational angle, $H$ is the vector component of the magnetic field either parallel or anti-parallel to the direction of light propagation, $d$ is the thickness of the crystal, and $V$ is the so-called Verdet constant, which is specific to the crystal's material. Therefore, a FRM with a crystal with known thickness and Verdet constant would allow to infer the strength of the magnetic field from the measurement of the rotational angle. In the considered application the setup needs to be adapted for usage from one side which is why a mirror-coated crystal is used. Thus, the light passes the crystal twice after which the rotational angle $\beta^{\dagger}$ is evaluated. Due to birefringence $\beta^{\dagger}$ is doubled compared to $\beta$.

However, instead of measuring the rotational angle directly, the change in intensity of the light is evaluated after it passes a set of polarisation filters. Initially unpolarised light passes the first filter - the polariser -, is rotated in the crystal, and passes the second filter - the analyser. The analyser is set at a relative angle $\alpha$ to the polariser. In order to calculate the light's intensity $I_{\alpha, \beta^{\dagger}}$ after the analyser for an intensity $I_{0}$ after the polariser, Malus' law can be applied. As in the proposed setup $I_{0}$ itself is never measured, it is more convenient to express $I_{\alpha, \beta^{\dagger}}$ relative to the intensity $I_{\alpha}:=I_{\alpha, \beta^{\dagger}=0}$ recorded at zero external magnetic excitation. Malus' law then states ${ }^{3}$ that

$$
I_{\alpha, \beta}=I_{0} \cdot \cos ^{2}\left(\alpha+\beta^{\dagger}\right)=I_{\alpha} \cdot \frac{\cos ^{2}\left(\alpha+\beta^{\dagger}\right)}{\cos ^{2}(\alpha)} .
$$

\section{CHARACTERISATION SETUP}

The setup according to Fig. 1 was built in order to facilitate the evaluation of the limitations regarding amplitude, spatial, and temporal resolution caused by the different components. This setup is largely similar to the one described in Sec. 1 with two noticeable exceptions. First, a beam splitter seperates the light path coming from the LED into a measurement path and a reference path. While the measurement path remains unchanged in principle, the intensity in the reference path is recorded using a photodiode. Secondly, the magnetic probe is replaced by Helmholtz coils. These allow the comparatively easy adjustment of the excitation field at the crystal by simply controlling the current passing through them. Furthermore, the magnetic field can be regarded as largely homogenous within the whole crystal. Lastly, it has to be mentioned that for the purposes of this characterisation the speed at which the setup is able to acquire data is secondary and has mostly been neglected. If necessary however, it can be extrapolated out of the given data. 


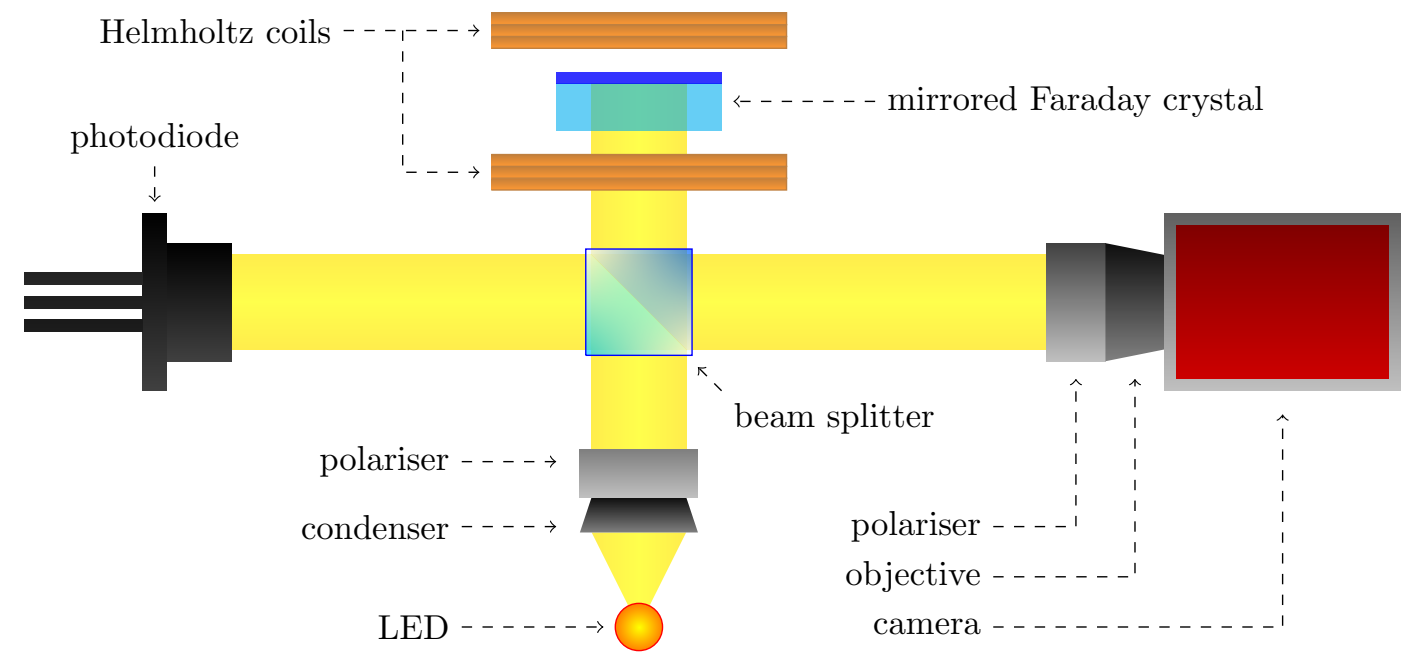

Figure 1: Characterisation setup of Faraday Rotation Magnetometer (elements not to scale).

The characterisation setup was built around a rare-earth substituted bismuth iron-garnet Faraday crystal produced by Matesy. Here, the Faraday-active layer is $5 \mu \mathrm{m}$ thick and deposited on one side of a glass block, while the other side has a mirror coating. The crystal's specified working range is around $\pm 2 \mathrm{kA} \mathrm{m}^{-1}$ or $\pm 4^{\circ}$. However, the most prominent feature of this crystal is its extraordinarily high Verdet constant of around $2.5 \times 10^{6} \mathrm{rad} \mathrm{T}^{-1} \mathrm{~m}^{-1}$. In comparison, this is four orders of magnitude higher ${ }^{2,4}$ than those of typically used crystals like terbium gallium garnet.

The light source is an Engin LZ1-00A102 high power LED with a maximum dominant wavelength of $590 \mathrm{~nm}$ matching the crystal's peak sensitivity.

The camera is an Allied Vision Mako G223B with an amplitude resolution defined by its 8 Bit internal ADC, a spatial resolution of $5 \mu \mathrm{m} \times 5 \mu \mathrm{m}$, and a frame rate of $49.5 \mathrm{fps}$.

The polarisers are set at an $\alpha=45^{\circ}$ relative angle, which according to Malus' Law in Eq. 2 maximises the absolute sensitivity of the recorded intensity to a changing polarisation angle $\beta^{\dagger}$. Given the working range of $\beta_{\text {min,max }}^{\dagger}=\mp 4^{\circ}$ of the crystal the relative angle $\alpha$ can be used to calculate the intensity working range $\Delta I_{\mathrm{w}}$ incident at the camera. This results in $\Delta I_{\mathrm{w}} \in\left[I_{\min }, I_{\max }\right] \approx\left[\left(I_{45^{\circ}} \times 0.86\right),\left(I_{45^{\circ}} \times 1.14\right)\right]$. It has to be noted, that given the offset of the range's lower bound $I_{\min }$, the camera's maximum intensity range $\Delta I_{\mathrm{c}}$ cannot be used to its full potential. Considering its ADC, the camera is then - at best - able to resolve a single frame to $2.8 \%$ of the intensity working range $\Delta I_{\mathrm{w}}$ due to quantisation noise.

\section{SOURCES OF ERROR AND COUNTERMEASURES}

Following first measurements performed with the characterisation setup, three main sources for limitations in either amplitude, temporal, or spatial resolution were identified: the camera, the light source, and the Faraday crystal.

\subsection{Camera: Signal Noise}

The output signal of a single pixel displayed a very high dependency on noise taking up about $40 \%$ of the intensity working range $\Delta I_{\mathrm{w}}$. As the noise showed a close to gaussian behaviour a Moving Average Filter of length $N$ would reduce the signal's standard deviation by a factor of approximately $1 / \sqrt{N}$. However, this gain in amplitude resolution would come at the cost of temporal resolution as the recording time for a single frame in most use cases would rise by a factor of $N$. 


\subsection{Camera and LED: Signal Drift}

Additionally to the noise, the ensemble of camera and light source displayed a long-term time dependency in form of a signal drift as shown in Fig. 2. This was mainly attributed to temperature shifts affecting the LED's efficiency and emission wavelength as well as the camera's sensitivity. It is important to reiterate, that unwanted and unregarded changes in the camera's output - either due to actual intensity changes of the LED or due to the camera's altered sensitivity - directly translate into the calculated rotational angle $\beta^{\dagger}$ and therefore into erroneous assumptions about the magnetic field. Thus, it is imperative to take those changes into consideration and minimise their effect. Here, instead of controlling the temperatures of the LED and the camera, the signal itself was corrected in order to compensate for its long-term temporal dependencies. As for the drift caused by the LED, the output signal was adjusted by contrasting it with the photodiode's signal in the reference arm. This largely eliminated the output signal's long-term drift. A further - albeit minor - improvement was achieved by correction for the camera's sensitivity using its internal temperature sensor. The effect of both of these corrections are also shown in Fig.2.

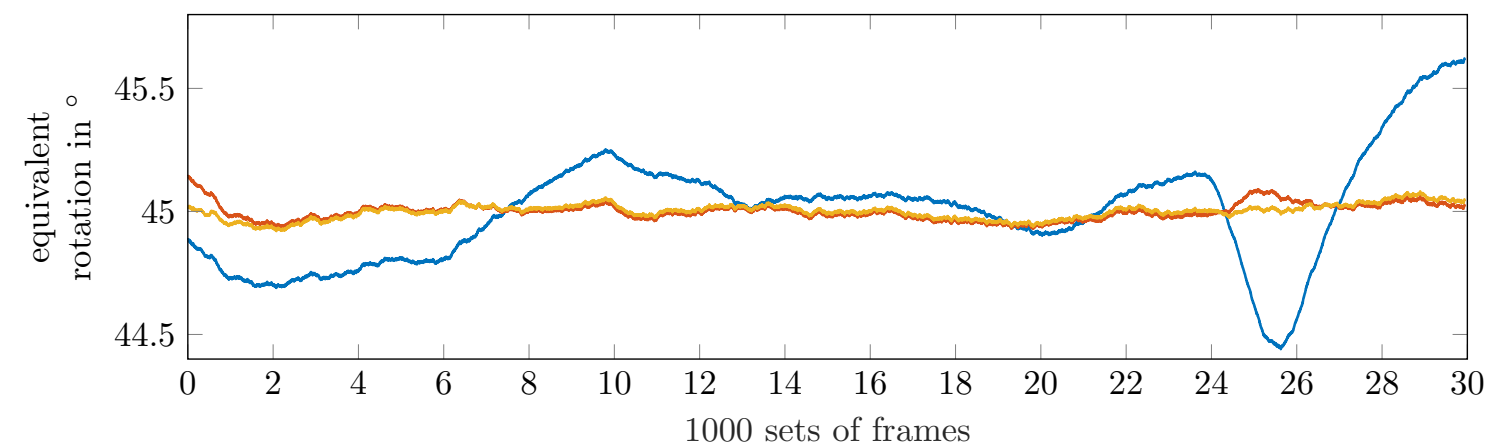

Figure 2: Long-term behaviour $(\approx 6$ hours $)$ of a single pixel's output signal using a Moving Average Filter with $N=1000$; blue/dark: without correction, red/medium: additional correction with photodiode's signal, orange/light: additional correction with camera temperature information

\subsection{Crystal: Spatial Behaviour}

At a close examination the crystal revealed some unexpected properties that need to be taken into consideration.

First, even within the specified working range of $\pm 2 \mathrm{kA} \mathrm{m}^{-1}$ there was a clear hysteresis in the recorded intensity or rather the equivalent rotation as can be seen in Fig. 3a. This of course would reduce the amplitude resolution of the infered magnetic field. However, as shown in Fig. 3b the effect of the hysteresis was negligible for a reduced working range of $\pm 1.5 \mathrm{kA} \mathrm{m}^{-1}$.

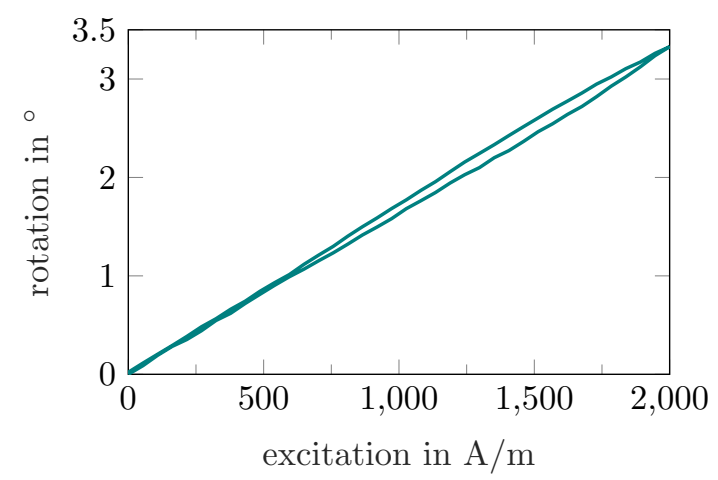

(a) specified working range

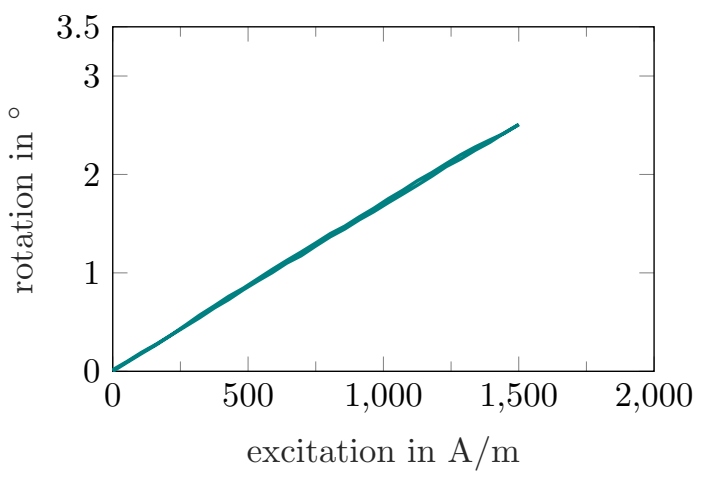

(b) reduced working range

Figure 3: Measurement of equivalent rotation within different working ranges w.r.t. magnetic excitation field 
Secondly, when viewed at a microscopic level the crystal exhibited ferrimagnetic behaviour. Figure 4 shows the rather regular ferrimagnetic pattern at an external homogenous excitation of $0 \mathrm{~A} \mathrm{~m}^{-1}$. The rate of repetition of a neighbouring pair of higher and lower rotation - corresponding to a pair of differently coloured stripes in Fig. 4 - can be interpreted as a spatial frequency. It is then possible to confirm the regularity with a two-dimensional spatial Fourier Transform.

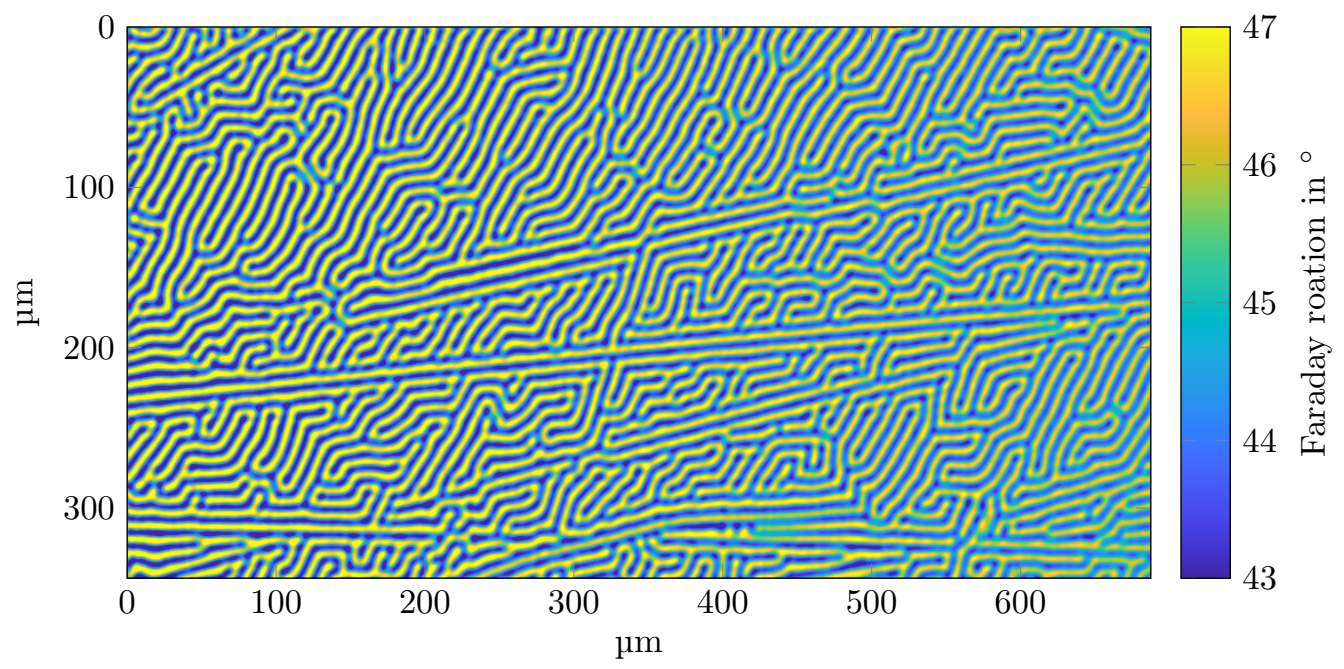

Figure 4: Ferrimagnetic pattern of crystal at $0 \mathrm{~A} / \mathrm{m}$

Now, when the excitation field changes the properties of the pattern change with it. As expected, there is a change in the average recorded intensity and therefore in the average equivalent rotation. Additionally however, the pattern structure changes as well. Mostly, the width of the stripes increases, i.e. the spatial frequency decreases. Furthermore, the regularity decreases, i.e. the spatial frequency spectrum becomes more blurred. Moreover, it does not seem possible to reliably associate a specific location on the crystal with a specific behaviour (relatively higher or lower rotation) even in the knowledge of the excitation field. In conclusion, due to the existence of the pattern, its dependence on the excitation field, and the lack of reproducibility of this dependence, the significance of the data collected from a single pixel is highly reduced. At any one time, it is only possible to infer a range of possible excitation fields from the range of rotations occurring in the pixel's vicinity. Slightly simplified this means that the ferrimagnetic pattern decreases the amplitude resolution for a single pixel. This is especially true for a non-homogenous excitation, which it would be in most sensible use cases.

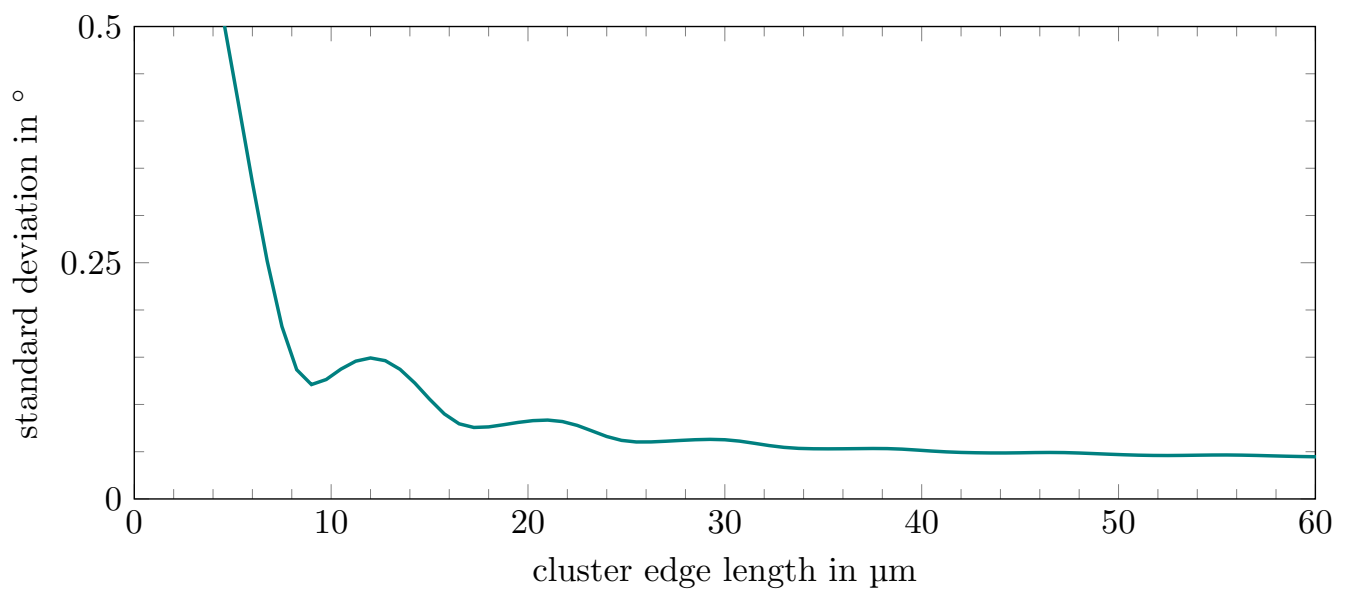

Figure 5: Standard deviation depending on cluster size at $0 \mathrm{~A} / \mathrm{m}$ 
Given the fairly high regularity of the pattern however, it is possible to reduce the negative impact of this ferrimagnetic behaviour on the amplitude resolution by evaluating a cluster of pixels rather than one. As shown in Fig. 5 the standard deviation of the mean value of a cluster as a function of its size takes the form of a dampened sinusoid. In the case of the used crystal the effect of the undulation becomes negligible at around $50 \mathrm{\mu m}$. This coincides with the examined pattern structures. On the other hand, this spatial averaging - while it may substantially increase the amplitude resolution - of course decreases the spatial resolution.

\section{SUMMARY}

The Faraday Rotation Magnetometer (FRM) shows promise for the non-destructive evaluation of magnetic microstructures. However, first setups only proved suitable for qualitative measurements. In order to quantify the limitations of the measurement principle with respect to amplitude, spatial, and temporal resolution we purpose-built a generalised form of the FRM for characterisation. We analysed it and identified the most important sources of error being the camera, the light source, and the Faraday crystal. We then proceeded by optimising the setup for use cases that require magnetically precise measurement, high acquisition speed, or

high spatial resolution. However, the signal enhancement with respect to amplitude resolution comes at the cost of temporal resolution or spatial resolution respectively. In both cases the correlation can easily be calculated. As temporal and spatial resolution seem to be uncorrelated, it is possible to define the amplitude resolution as a function of the other two. For any given set of requirements this enables us to give a first estimate of the feasibility of measuring with a FRM. Furthermore, this contribution may be used as a build and signal enhancement guideline whenever a FRM proves to be a suitable measurement device in principle.

\section{REFERENCES}

[1] Egger, J. and Zagar, B., "In-Line Signal Processing of Faraday-Magnetometer Scans," in [Proceedings of EUROCAST 2017], (2017).

[2] Tumanski, S., [Handbook of Magnetic Measurements], CRC Press Taylor \& Francis Group (2011).

[3] Pedrotti, F., Pedrotti, L., Bausch, W., and Schmidt, H., [Introduction to Optics], Pearson Education Company (1993 (second edition)).

[4] "Terbium Gallium Garnet - TGG." Northrop Grumman Synoptics, 2011 https://www.northropgrumman . com/BusinessVentures/SYNOPTICS/Products/SpecialtyCrystals/Documents/pageDocs/TGG.pdf. (2011). 